\title{
Recuerdo inmediato inverso de palabras en euskera altas y bajas en imagen, mediante la mnemotecnia de la palabra clave
}

\section{Inmediate productive recall of high- and low-image-value basque words with the keyword mnemonic}

\author{
Aitziber Goñi-Artola, María Ángeles González \\ Universidade da Coruña
}

\begin{abstract}
Resumen
Una modalidad de recuerdo que permite la aplicación de la mnemotecnia de la palabra clave es la tarea de recuerdo inverso o productivo. Este trabajo analizó si existían diferencias significativas en el recuerdo inverso inmediato de palabras en euskera altas y bajas en imagen. Un grupo de 103 estudiantes de $3^{\circ}$ y $4^{\circ}$ curso de Educación Secundaria Obligatoria aprendió el significado de 24 palabras en euskera, 12 con alto valor de imagen, y 12 bajas en imagen, mediante la mnemotecnia de la palabra clave. Se encontró que el recuerdo inmediato inverso de las palabras altas en imagen, era significativamente superior al recuerdo inmediato inverso de las palabras bajas en imagen.

Palabras clave: método de la palabra clave, mnemotecnia, memoria, imagen, recuerdo inverso.
\end{abstract}

\begin{abstract}
A modality of recall that the keyword mnemonic allows is the task of inverse or productive recall. This work analyzes differences between the immediate productive recall of basque words with high and low image value. A group of 103 students of 3rd and 4th year of Compulsory Secondary Education learned the meaning of 24 basque words, 12 with high image value, and 12 low in image, by means of the keyword mnemonic. We found that the immediate productive recall of the high image words was significantly superior to the immediate productive recall of the low image words.

Keywords: keyword mnemonic, mnemonics, memory, image, productive recall.
\end{abstract}

La mnemotecnia de la palabra clave (MPC) es una estrategia mnemotécnica que se utiliza principalmente para el aprendizaje del vocabulario de un segundo idioma. Esta estrategia fue presentada por Atkinson y Raugh en 1975 para el aprendizaje de vocabulario en ruso (Atkinson \& Raugh, 1975).

Esta estrategia consta de dos fases. En la primera fase asociamos la palabra que queremos aprender, por ejemplo, la palabra ESKU ("mano" en euskera), con una que suene parecido en nuestro idioma (esquí). Esta palabra de sonido parecido a la palabra que queremos aprender se denomina palabra clave, y es la que le da el nombre a la estrategia. En la segunda fase formamos una imagen mental interactiva que relacione la palabra de sonido parecido (esquí) con la traducción en nuestro idioma de la palabra que queremos aprender (mano).

A la hora de estudiar la eficacia de la mnemotecnia de la palabra clave, habitualmente se ha optado por una tarea de recuerdo receptivo, esto es, se presenta la palabra en el segundo idioma y el sujeto debe facilitar la traducción en su idioma. Este tipo de recuerdo está más acorde con el método de aprendizaje, ya que ambos siguen la misma ruta (primero buscamos la palabra que suena parecido y luego buscamos la imagen mental). Pero cuando queremos dominar un idioma, también es necesaria la tarea inversa, es decir, que desde la palabra en nuestro idioma seamos capaces de dar la traducción en el segundo idioma (siguiendo con el ejemplo anterior, ver la palabra "mano" y recordar "esku"). A este tipo de recuerdo se la llama recuerdo productivo o inverso.

Pressley, Levin, Hall, Miller y Berry (1980) analizaron si existían diferencias a la hora de recordar de modo receptivo o de modo inverso cuando se utilizaba la mnemotecnia de la palabra clave (MPC). Cuando el recuerdo era receptivo, quienes aprendían utilizando la MPC obtuvieron mejor resultado que los que aprendieron solamente por repetición, o que aquellos a los que no se instó a utilizar ningún método. En recuerdo inverso, no hubo diferencias significativas entre los grupos experimentales.

Pressley y Levin (1981) utilizaron un enfoque diferente a la hora de abordar la eficacia de la MPC. Emplearon palabras de uso común, que estaban previamente bien integradas en la memoria de los participantes, y la dificultad residía en las definiciones que debían aprender, que eran poco frecuentes. Cuando el recuerdo era inverso, la ejecución fue superior en el grupo experimental (MPC) que en el grupo control.

El recuerdo es superior cuando éste es receptivo que cuando es productivo (inverso), porque este último es una tarea más exigente (Ellis \& Beaton, 1993). Estos investigadores observaron que cuando el recuerdo era productivo, los sujetos que aprendían por repetición recordaban significativamente más palabras que los grupos mnemónicos o los que utilizaron su propia estrategia. Ellis y Beaton (1993) atribuyen esta dificultad 
principalmente a tres factores. Uno, el recuerdo productivo requiere la producción de nuevos patrones, ya sean de ortografía o de pronunciación. Dos, los sujetos tienen más práctica a la hora de recordar de modo receptivo que de modo productivo. $\mathrm{Y}$ tres, la nueva palabra que queremos aprender en otro idioma solo está asociada con su equivalente en el idioma nativo, no tiene otras asociaciones en el sistema léxico.

Gruneberg y Pascoe (1996) aplicaron tres criterios de corrección para el recuerdo productivo. En el criterio estricto, la palabra en el idioma extranjero debe ser totalmente correcta, en el criterio medio casi correctas, pero no estrictamente fonéticamente correcta, y en el criterio blando solo sustancialmente correcto. Cuando se aplicaba un criterio estricto de corrección, no había diferencias entre el grupo experimental (MPC) y el grupo control. En cambio, cuando el criterio era blando los sujetos que aprendieron a través de la MPC recordaron significativamente más palabras que el grupo control.

Campos, Amor y González (2003) analizaron la eficacia de la MPC en el recuerdo inverso, con palabras altas y bajas en imagen, con listas cortas y listas largas y con diferentes formas de generar la palabra clave. Se analizaron tres formas de presentar la palabra clave, presentada por el investigador, generada por un grupo de compañeros o generada por los propios sujetos. La MPC se comparó con el aprendizaje por repetición. Los sujetos que aprendieron a través de la MPC con las palabras clave generadas por compañeros, recordaron significativamente menos palabras altas en imagen que los demás grupos. Con palabras bajas en imagen sucedió algo similar. El grupo que aprendió a través de MPC con palabras clave generadas por los compañeros, recordó significativamente menos que el grupo de repetición y el grupo que aprendió a través de MPC y generó su propia palabra clave. El grupo que generó su propia palabra clave también recordó significativamente más palabras bajas en imagen de modo inverso, que el grupo en el que la palabra clave fue facilitada por el experimentador. Esto podría deberse a que se establece una buena asociación entre la palabra a aprender y la palabra clave (Campos et al., 2003).

Con listas largas de palabras (32 palabras), Campos et al. (2003) no encontraron diferencias significativas en el recuerdo, independientemente de la estrategia de aprendizaje empleada (repetición, palabra clave generada por compañeros, palabra clave generada por experimentador, y palabra clave generada por los propios sujetos). Al reforzar con dibujos la estrategia de aprendizaje, tampoco se encontraron diferencias significativas en el recuerdo inverso.

Beaton, Gurember, Hyde y Sufflebottom (2005) quisieron examinar el papel que la "calidad" de la imagen tenía en el recuerdo receptivo y productivo. Primero compararon la eficacia de la MPC frente al aprendizaje por repetición. El recuerdo de los que utilizaron la MPC fue significativamente superior, tanto el recuerdo receptivo como el productivo o inverso. También vieron que cuando se utilizaba la MPC con imágenes de "buena calidad", el recuerdo tanto receptivo como productivo era significativamente superior. Pero cuando la calidad de la imagen era "pobre", no existían diferencias entre los que utilizaron la MPC y los que aprendieron por repetición, ni en el recuerdo receptivo ni en el productivo. Los autores concluyen que cuando la calidad de las imágenes es suficientemente buena, el recuerdo tanto inverso como receptivo mejora.

Campos, Rodríguez-Pinal y Pérez-Fabello (2013; 2014) encontraron que el recuerdo inverso de los participantes que utilizaron la MPC fue superior al de los sujetos que aprendieron por repetición, y que los sujetos con alta capacidad de manipulación o control de imagen recordaban más palabras cuando el recuerdo era inverso que los participantes bajos en control de imagen.

El objetivo de este trabajo es analizar si existen diferencias significativas en el recuerdo inmediato inverso de palabras en euskera altas y bajas en imagen, cuando se utiliza la mnemotecnia de la palabra clave.

\section{Método}

\section{Participantes}

En la investigación participó un grupo de 103 estudiantes de Educación Secundaria Obligatoria de varios institutos de A Coruña, 50 hombres y 53 mujeres, con una media de edad de 14.94 años, una desviación típica de 1.07, y un rango entre 13 y 18 años.

\section{Material}

Utilizamos una lista de 24 palabras en euskera (12 altas en imagen y 12 bajas en imagen) y su correspondiente traducción al castellano. También 24 palabras clave para cada una de las palabras en euskera, y 24 frases interactivas relacionando la palabra clave con la correspondiente traducción al castellano de la palabra en euskera, seleccionadas a partir de las publicadas por Valle (1998), cuyas palabras clave y frases interactivas fueron generadas por un grupo de compañeros de similares características a los estudiantes del estudio.

Se consideran altas en imagen las palabras con un valor de imagen mayor que 5.20, y palabras bajas en imagen las que tienen un valor menor de 4.21 (Valle, 1998). El conjunto inicial de sustantivos traducido al euskera, se redujo al rechazar las palabras que sonasen o escribiesen de forma similar en castellano y en euskera. Se seleccionaron al azar 16 palabras altas en imagen y 16 palabras bajas en imagen (32 sustantivos en total). Esas 32 palabras en euskera, y sus traducciones, fueron presentadas a un grupo de estudiantes de características similares a los participantes, 80 alumnos de $3^{\circ}$ y $4^{\circ}$ de E.S.O. de la ciudad de A Coruña. Dichos estudiantes elaboraron, para cada palabra en euskera, una palabra clave y una frase que describiera una imagen mental en la que interactuasen la palabra clave con el significado de la palabra en euskera. De todas las palabras clave que generó este grupo de compañeros, se eligieron las más frecuentes. Para el presente estudio se utilizaron 24 de esas palabras en euskera (12 altas en imagen y 12 bajas en imagen). Una vez seleccionadas también las palabras clave para estos 24 sustantivos en euskera, se escogieron las frases interactivas que aparecían con mayor frecuencia. 


\section{Procedimiento}

Al grupo de participantes, en sus respectivas clases, se les presentó, en diapositivas y a través de un proyector, la lista de 24 palabras en euskera, para que aprendieran su traducción al castellano mediante la mnemotecnia de la palabra clave. De estas palabras, cuyo orden de presentación se realizó al azar, 12 tenían alto valor de imagen, y las otras 12, bajo valor de imagen. Cada diapositiva constaba de la palabra en euskera, de su correspondiente traducción al español, de la palabra clave y de una frase interactiva correspondiente. Como se ha descrito en el apartado de material, estas palabras clave, y sus correspondientes frases interactivas, las habían generado previamente un grupo de compañeros de características similares a los participantes de este estudio. Cada diapositiva permanecía 15 segundos en pantalla y cambiaba de forma automática a la siguiente. Una vez concluida la fase de aprendizaje, a los participantes se les aplicó la prueba de recuerdo inverso.

\section{Resultados}

Con el fin de averiguar si existían diferencias significativas en el recuerdo inmediato inverso de palabras en euskera altas y bajas en imagen, se efectuó una prueba $t$ para muestras relacionadas, tomando como variable dependiente la puntuación de las palabras Altas y Bajas en imagen que obtuvieron los participantes en la prueba de recuerdo inmediato inverso. Las medias y desviaciones típicas del recuerdo inmediato inverso de las palabras Altas y Bajas en imagen, figuran en la Tabla 1 .

Tabla 1.

Medias y Desviaciones Típicas del Recuerdo Inmediato Inverso Obtenido por los Participantes en las Palabras Altas y Bajas en imagen

\begin{tabular}{lcc}
\hline & \multicolumn{2}{c}{ Recuerdo Inmediato Inverso } \\
\cline { 2 - 3 } Grupos & $M$ & $S D$ \\
\hline Palabras Altas en imagen & 1.96 & 1.97 \\
Palabras Bajas en Imagen & 1.04 & 1.35 \\
Total & 3.00 & 2.86 \\
\hline
\end{tabular}

Encontramos que la puntuación que obtuvieron los estudiantes en recuerdo inmediato inverso de las palabras altas en imagen $(M=1.96 ; S D=1.97)$, era significativamente superior a la puntuación en recuerdo inmediato inverso de las palabras bajas en imagen $(M=$ $1.04 ; S D=1.35), t(102)=5.21, p<.001$.

\section{Discusión}

Se ha encontrado que la puntuación que obtuvieron los estudiantes en recuerdo inmediato inverso de palabras en euskera altas en imagen, era superior a la puntuación en recuerdo inverso de palabras de este idioma bajas en imagen. Estos resultados coinciden con los obtenidos por Campos et al. (2003) en listas cortas de palabras de un segundo idioma (en ese trabajo, 16 palabras en latín), aunque con listas largas (32 palabras) estos autores no encontraron diferencias en el recuerdo inverso de palabras altas y bajas en imagen. En nuestra investigación, el número de palabras empleadas (24 palabras) se encuentra en un punto medio, por lo que cabe pensar que el número de palabras a aprender influye en el recuerdo inverso de palabras altas y bajas en imagen.

Vemos que el valor de imagen de las palabras es una variable a tener en cuenta en el recuerdo inverso, al menos cuando el material a aprender son palabras de un segundo idioma. Creemos que es necesario seguir investigando en esta línea, en este y otros idiomas, y con listas de diferente tamaño, para contribuir a mejorar el rendimiento de los alumnos a la hora de aprender a través de la MPC.

\section{Referencias}

Atkinson, R. C. \& Raugh, M. R. (1975). An application of the mnemonic keyword method to the acquisition of a Russian vocabulary. Journal of experimental psychology: Human learning and memory, 1, 126-133. http://dx.doi.org/10.1037/0278-7393.1.2.126.

Beaton, A., Gruneberg, M., Hyde, C., Shufflebottom, A. \& Sykes, R. (2005). Facilitation of receptive and productive foreign vocabulary learning using the keyword method: The role of image quality. Memory, 13, 458-471. http://dx.doi.org/10.1080/09658210444000395.

Campos, A., Amor, Á. \& González, M. Á. (2003). ¿Es eficaz la mnemotecnia de la palabra clave en el recuerdo inverso? Revista Galego-Portuguesa de Psicoloxía e Educación, 7(9), 47-57.

Campos, A., Rodríguez-Pinal, M. D. \& Pérez-Fabello, M. J. (2013). Aprendizaje del idioma gallego mediante la mnemotecnia de la palabra clave, en personas bilingües, altas y bajas en control de imagen. Revista de Investigación en Educación, 11, 50-59.

Campos, A., Rodríguez-Pinal, M. D. \& Pérez-Fabello, M. J. (2014). Receptive and productive recall with the keyword mnemonics in bilingual students. Current Psychology, 33, 64-72. http://dx.doi.org/10.1007/s12144-013-9197-y.

Ellis, N. \& Beaton, A. (1993). Factors affecting the learning of foreign language vocabulary: Imagery keyword mediators and phonological short-term memory. The Quarterly Journal of Experimental Psychology, 46, 533-558. http://dx.doi.org/10.1080/14640749308401062.

Gruneberg, M. M. \& Pascoe, K. (1996). The effectiveness of the keyword method for receptive and productive foreign vocabulary learning in the elderly. Contemporary Educational Psychology, 21, 102-109. http://dx.doi.org/10.1006/ceps.1996.0009.

Pressley, M. \& Levin, J. R. (1981). The keyword method and recall of vocabulary words from definitions. Journal of Experimental Psychology: Human Learning and Memory, 7, 72-76. http://dx.doi.org/10.1037/0278-7393.7.1.72.

Pressley, M., Levin, J. R., Hall, J. W., Miller, G. E. \& Berry, J. K. (1980). The keyword method and foreign word acquisition. Journal of Experimental Psychology: Human Learning and Memory, 6, 163-175.

http://dx.doi.org/10.1037/0278-7393.6.2.163. 
Valle, F. (1998). Normas de imaginabilidad. Oviedo: Servicio de Publicaciones de la Universidad de Oviedo. 